\title{
FRACTURE TOUGHNESS OF DIFFERENT ADHESIVE/DENTIN INTERFACES ANALYZED BY FINITE ELEMENT STRESS ANALYSIS
}

\author{
Hend Abdelfattah Mustafa*, Mohamed Mahmoud kandil ${ }^{* *}$, \\ Tamer Mounir Nassef ${ }^{* * *}$ and Tarek Salah ${ }^{* * * *}$
}

\begin{abstract}
Objectives: This study aimed to assess the fracture toughness of different dentin adhesive approaches and to evaluate the stress distribution at the bond interface during fracture toughness testing by finite element analysis.

Materials and Methods: Specimens for chevron-notched beam fracture toughness (CNB) were prepared with dimensions of $(3.0 \mathrm{~mm} \times 4.0 \mathrm{~mm} \times 20 \mathrm{~mm})$ according to ISO 24370:2005 standard. Chevron notch was prepared at the adhesive-dentin interface using a highly polished stainless steel spacer. Four different dentin adhesives approaches were used total etch 3 and 2 step and self adhesives 2 and 1 step. A total of 60 specimens were prepared for each adhesive approach $(n=15)$. Each specimen was loaded until failure in a 4-point bend test setup and the fracture toughness was calculated according to the ISO specifications. Finite element modeling of the specimens was carried out in order to analyze stress distribution during fracture toughness testing. Data were analyzed by one-way ANOVA followed by tukey's post hoc test with significant level at $\mathrm{p}<0.001$ level $(\mathrm{p}=0.0001)$.
\end{abstract}

Results: Solobond plus 3-step etch-and-rinse showed the highest mean fracture toughness (1.78 $\left.\mathrm{MPam}^{1 / 2}\right)$ Whereas, Futurabond M+ 1-step self-etch showed the lowest value $\left(1.13 \mathrm{MPam}^{1 / 2}\right)$. These finding were supported by finite element analysis which showed stresses to concentrate at the adhesive-dentin interface during fracture toughness loading and crack propagation.

Conclusion: The adhesive approach has a strong effect on the fracture toughness of the adhesive-dentin interface. the 3-step etch-and-rinse approach showed the highest mean fracture toughness while 1-step self-etch approach showed the lowest value.

KEYWORDS: Fracture toughness, CNB, Adhesives, Dentin, Finite element analysis.

\footnotetext{
* Faculty of Dentistry Ain-Shams University

** Lecturer of Dental Materials, Faculty of Dentistry, Ain-Shams University, Cairo, Egypt

*** Associate Professor, Computer and Software Engineering, Misr University for Science and Technology,

*** Professor, Dental materials Department, Faculty of Dentistry, Ain- Shams University
} 


\section{INTRODUCTION}

Recent years have seen rapid advances in dental adhesive technology. Manufacturers continue to release new adhesive systems with improved composition and manipulation. The longevity of a restoration can be predicted to some extend by its adhesive ability which can be evaluated by bond strength. ${ }^{(1)}$

Bond strength testing is the most common worldwide method used to assess the efficacy of bonding to enamel and dentin used to evaluate adhesive systems and predict the clinical performance and the durability of dental restorations, so the bond strength tests must be accurate and clinically reliable..$^{(2)}$

An ideal bond strength test should be precise, and not technique-sensitive. It should involve the use of fairly unsophisticated and inexpensive test protocols. Oilo et al. classified the bond strength tests into qualitative screening tests which study bond failure, and quantitative tests predict the load capacity and the bond lifetime. ${ }^{(3)}$

Dental adhesives are usually tested in shear or tension despite the fact that neither of these testing approaches measures the local stress triggering failure. Because the stress level varies extensively over the bonded surface, the validity of these bond strength tests is questionable due to the name as, shear or tensile may not reflect to the true and complete stress situation. As these tests assume uniform shear or uniaxial tensile conditions. They can be measured using a macro or micro test set-up depending on the size of the bonded area. ${ }^{(4)}$

There are several inconsistencies regarded the reported bond strengths that could lead to misinterpretation of the data about the bonding functionality of different adhesives. Several variables could affect the obtained bond strength data these include the sample geometry and size, applied load and inherent material properties of the various adhesives interfaces. ${ }^{(5)}$
This makes it challenging for the clinician to determine the most appropriate adhesive for a given procedure and for the adhesive manufacturer to optimize its composition. As a result, an adhesion test protocol based on the fracture mechanics was proposed to generate data by separation of the effect of composition from that of the joint geometry on the shear and tensile bond strengths. ${ }^{(6)}$

Fracture toughness is an intrinsic property of a material and is the measure of a materials resistance to crack propagation. Since fracture toughness test could be an effective method for characterization of the intrinsic fracture resistance and the dentinadhesive-interface durability. ${ }^{(7)}$

Finite Elements Analysis is a numerical analysis tool that allows the simulation of experimental situations and to analyze and solve complex problems in the biomechanical area. ${ }^{(8)}$

This study was designed to assess the effect of different adhesive approaches on the fracture toughness of different dentin adhesive interfaces and to analyze the stress distribution at this interface. Assessment of the fracture toughness was done experimentally using CNB supplemented by finite element analysis.

\section{MATERIALS AND METHODS}

\section{Materials used in study}

The materials used in the study and their description are shown in (Table 1).

\section{Specimens Preparation and Material Testing.}

The fracture toughness of adhesive-dentin interfaces was measured using a chevron-notched beam (CNB) test, adapted from the ISO 24370 standard to measure fracture toughness of fine ceramics. ${ }^{(9)}$

Specimens were divided into four groups $(n=15)$ according to the type of adhesives and the technique of the adhesive systems application as shown in (Figure 1). 
TABLE (1): Materials used, their brand name and manufacturer, main constituents and lot number.

\begin{tabular}{|c|c|c|c|}
\hline Materials & $\begin{array}{l}\text { Brand Name \& } \\
\text { Manufacturer }\end{array}$ & Main Constituents & Lot no. \\
\hline Acid etchant & $\begin{array}{l}\text { DENTSPLY DeTrey } \\
\text { Conditioner } 36^{\mathrm{TM}} \\
\text { DENTSPLY, Germany }\end{array}$ & $36 \%$ phosphoric acid gel & $\leftarrow \operatorname{lot} 1708001$ \\
\hline $\begin{array}{l}\text { Universal adhesive } \\
\text { Set } 4 \mathrm{ml} \text { primer, } 4 \mathrm{ml} \\
\text { adhesive }\end{array}$ & $\begin{array}{l}\text { Solobond Plus }{ }^{\mathrm{TM}} \text { Voco } \\
\text { GmbH, Cuxhaven, } \\
\text { Germany }\end{array}$ & $\begin{array}{l}\text { \&Primer: water, acetone, hydroxymethacrylate, } \\
\text { fluorides, acidic monomers, maleic acid } \\
\leftarrow \text { Adhesive: acetone, BIS-GMA, TEGDMA, } \\
\text { hydroxymethacrylate, }\end{array}$ & $\leftarrow \operatorname{lot} 1841533$ \\
\hline $\begin{array}{l}\text { Universal adhesive } \\
\text { Bottle } 5 \mathrm{ml}\end{array}$ & $\begin{array}{l}\text { Futurabond } \mathrm{M}^{+\mathrm{TM}} \text { Voco } \\
\text { GmbH, Cuxhaven, } \\
\text { Germany. }\end{array}$ & $\begin{array}{l}\leftarrow \text { Bisphenol A diglycidylmethacrylate, Acidicadhesive } \\
\text { monomer (10-MDP), UDMA, ethanol, acidic } \\
\text { adhesive monomer, catalyst }\end{array}$ & $\leftarrow$ lot 1828488 \\
\hline $\begin{array}{l}\text { Universal nanohybrid } \\
\text { resin composite }\end{array}$ & $\begin{array}{l}\text { Grandio }^{\mathrm{TM}} \text { A2Voco } \\
\text { GmbH, Cuxhaven, } \\
\text { Germany }\end{array}$ & $\begin{array}{l}\leftarrow \text { Bis-GMA, TEDGMA, UDMA matrix. } \\
\leftarrow 87 \text { wt. } \% / 71.4 \text { Vol. } \% \text { inorganic filler loading. }\end{array}$ & $\leftarrow \operatorname{lot} 1808086$ \\
\hline
\end{tabular}

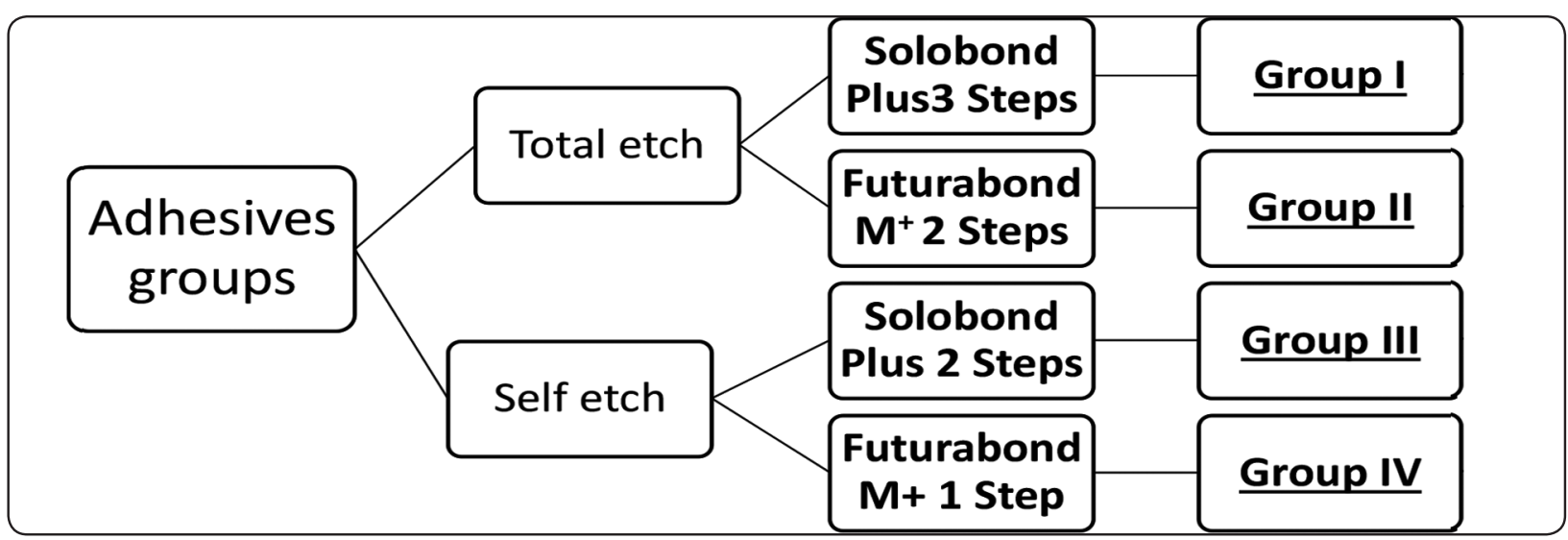

Fig. (1): Flow chart showing specimens' grouping for each test.

Freshly extracted non-carious human wisdom teeth were stored in distilled water at $4{ }^{\circ} \mathrm{C}$ and used within one month after extraction. Beams of dentin were prepared using an automated diamond saw with a cooling water system. (IsoMet 4000, Buehler Ltd., Lake Bluff, IL, USA) The occlusal surface was flattened perpendicular to the long axis of the tooth exposing dentin surfaces. Beam with approximately dimension $4 \mathrm{~mm}$ thickness $\times 3 \mathrm{~mm}$ width $\times 10 \mathrm{~mm}$ length were cut longitudinaly. A rectangle split teflon mold $4 \pm 0.20 \mathrm{~mm}$ thickness, $3 \pm 0.20 \mathrm{~mm}$ width and $20 \mathrm{~mm}$ long, with a corresponding metal ring was used to produce pre-notched rectangular stick specimens.
Chevron spacer was made of highly polished stainless steel with dimensions $3.8 \mathrm{~mm}$ length and 0.30 $\mathrm{mm}$ thickness with chevron tip dimension $0.80 \pm 0.8$.

A celluloid strip (Stripmat, POLYDENTIA, CH6805 Mezzovico, Switzerland) was placed on a flat glass slab, the teflon mold was placed on top of it. Then the prepared dentin beam was inserted into the teflon mold and the spacer was then placed against the dentin substrate half specimen to delineate the chevron-shaped notch.

Exposed dentin around the spacer was then treated with the proprietary adhesives as recommended by the manufacturers as shown in (Table 2). 
TABLE (2): Bonding steps for the different groups.

\begin{tabular}{|c|c|}
\hline Groups & Bonding Steps \\
\hline Group I & $\begin{array}{l}\text { Apply etching gel for } 10 \mathrm{~s} \text {, water rinsing, air drying. } \\
\text { Apply Primer for } 10 \mathrm{~s} \text {; air-dry. } \\
\text { Apply Bond for } 10 \mathrm{~s} \text {, air thinning and light cure. } \\
\text { Apply composite and light cure. }\end{array}$ \\
\hline Group II & $\begin{array}{l}\text { Apply etching gel for } 10 \mathrm{~s} \text {, water rinsing, air drying. } \\
\text { Apply bond for } 20 \mathrm{~s} \text {, air thinning and light cure. } \\
\text { Apply composite and light cure. }\end{array}$ \\
\hline Group III & $\begin{array}{l}\text { Apply Primer for } 20 \mathrm{~s} \text {; air-dry. } \\
\text { Apply Bond for } 10 \mathrm{~s} \text {, air thinning and light cure. } \\
\text { Apply composite and light cure. }\end{array}$ \\
\hline Group IV & $\begin{array}{l}\text { Apply Bond for } 20 \mathrm{~s} \text {, air thinning and light cure. } \\
\text { Apply composite and light cure. }\end{array}$ \\
\hline
\end{tabular}

Packing of composite increments of approximately $1 \mathrm{~mm}$ thickness into the rest of the mold was performed. Each increment was light cured for 30 $\mathrm{s}$, the last increment was flattened using a glass slab and and light cured for 30 seconds using the LED curing light (3M ESPE, Germany) after covering with a celluloid strip (Stripmat, POLYDENTIA, CH-6805 Mezzovico, Switzerland) according to the manufacturers' directions.

The specimen was then removed from the mold and was cured from all sides for $10 \mathrm{~s}$ for each side to ensure complete polymerization.

All specimens dimensions were checked with a digital caliper (Mitutoyo digital caliper, Japan).

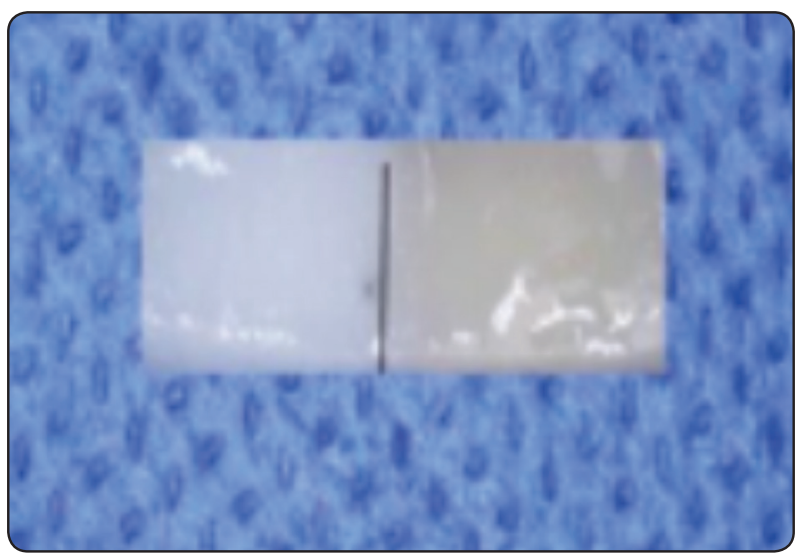

Fig. (2): Chevron-notched beam fracture toughness speciemen.

\section{Assessment of the fracture toughness value of dif- ferent adhesives-dentin interfaces experimentally.}

Each specimen was transferred to the universal testing machine (Model 3345; Instron Industrial Products, Norwood, MA, USA) tested in a 4-point bend test setup with a crosshead speed of $0.2 \mathrm{~mm} /$ min. The outer and inner span was 13 and $6.5 \mathrm{~mm}$ respectively.

Each specimen was supported on two parallel stainless steel rods apart with the notch centrally located on the tensile side. Load was applied until failure of the specimen. (Figure 3).

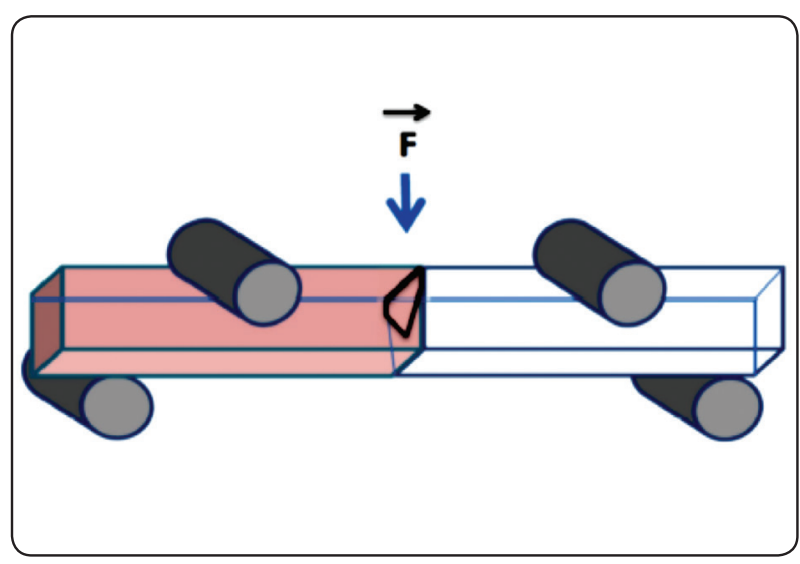

Fig. (3): Showing 4-point bend test set-up. 
The fracture toughness value was calculated as follows:

Calculation of fracture toughness value $\mathrm{K}_{\mathrm{I}, \mathrm{CNB}}$

$$
K \mathrm{I} c=\frac{F(S 0-S i)}{B W^{3 / 2}} x \frac{Y * \min }{\sqrt{1000}}
$$

Where: $\mathrm{KIc}=$ the fracture toughness value mode I in MPam1/2, F = the total force in Newton, $\mathrm{So}=$ the outer span in $\mathrm{mm} 6.5 \mathrm{~mm}, \mathrm{Si}=$ the inner span in $\mathrm{mm} 13 \mathrm{~mm}, \mathrm{~B}=$ test specimen thickness in $\mathrm{mm}(3.00$ $\pm 0.2 \mathrm{~mm}), \mathrm{W}=$ test specimen width in $\mathrm{mm}(4.00$ $\pm 0.2 \mathrm{~mm}), \mathrm{Lo}=$ chevron tip dimension in $\mathrm{mm}(0.8$ $\mathrm{mm}), \mathrm{L} 1=$ the position of the bottom part in $\mathrm{mm}$ $(3.8 \mathrm{~mm})$, and $\mathrm{Y}^{*} \mathrm{~min}=$ the minimum stress intensity factor coefficient dimensionless. and calculated from the following function:

$Y^{*} \min =\left[2.92+4.52\left(\frac{L 0}{W}\right)+10.14\left(\frac{L 0}{W}\right)^{2}\right] \sqrt{\frac{\left(\frac{L 1}{W}\right)-\left(\frac{L 0}{W}\right)}{1-\left(\frac{L 0}{W}\right)}}$

\section{Finite element analysis of the fracture toughness} experimental models.

The process of finite element analysis was carried out using modeling software (SolidWorks 2018, SolidWorks Corporation, Concord, MA, USA). It included three phases: the pre-processing, processing and post-processing phases.

In the pre-processing phase, finite element models configuration were based on the experimental fracture toughness test set-up, consisting of 4 linear 3D finite element micro-models of dentin restored with composite resin with dimensions $3 \mathrm{~mm}$ width $\times 4 \mathrm{~mm}$ thickness $\times 20 \mathrm{~mm}$ length and with initial crack sizes also the same as those used in the experimental study with elastic modulus based on the data obtained from previous studies. ${ }^{(10,11)}$ The meshing of the models was done using the Computer Aided Design programs CAD.

It was presumed that the characteristics of all materials used in this study were isotropic, homogenous, and linear for mechanical point of view. Each material's dimensions and characteristics (elasticity module and Poisson ratio) based on data from previous studies were gathered and uploaded to the software. (11)

The modulus of elasticity (in gegapascals) and poisson's ratio of dentin, composite and the tested adhesives materials were determined from previous literatures.(12-14) and are shown in (Tables 3, 4).

TABLE (3): The modulus of elasticity and poisson's ratio of used materials.

\begin{tabular}{|c|c|c|}
\hline Materials & Modulus (GPa) & Poisson's ratio \\
\hline Dentin & $\mathbf{1 7 . 4}$ & $\mathbf{0 . 3 1}$ \\
\hline Composite & $\mathbf{1 6 . 6}$ & $\mathbf{0 . 3 1}$ \\
\hline
\end{tabular}

For each adhesive system, the finite element models differed in the dimensions and morphological characteristics of the dentin-adhesive interface, they were simulated as shown (Figure 4).

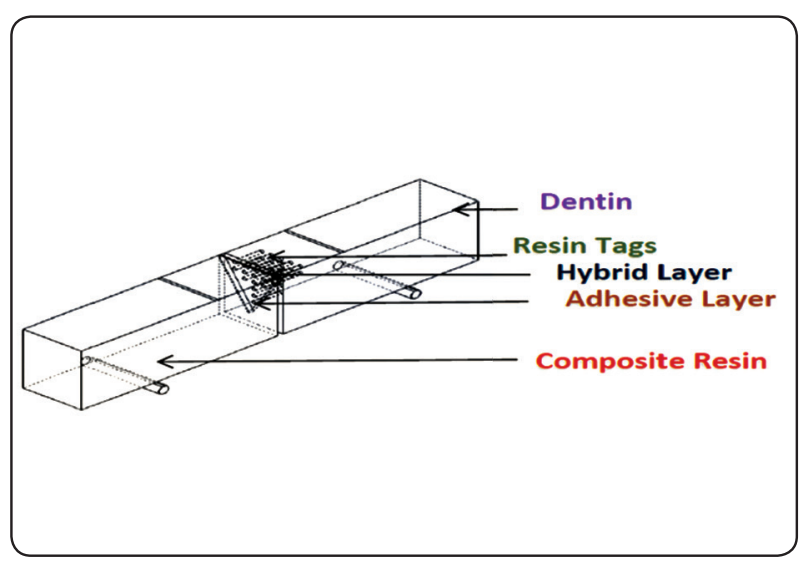

Fig. (4): The finite element model showing different simulated structures

In the processing phase, The contact interfaces between each part of the model (dentin-adhesive composite interface or cement) were assumed to be fully bonded; as such, no sliding was allowed in $\mathrm{X}, \mathrm{Y}$, and $\mathrm{Z}$ directions. Boundary conditions were set according to the mechanical testing conditions. 
TABLE (4): Mechanical and geometrical properties of the dentin-adhesive interface components for each approaches.

\begin{tabular}{|c|l|c|c|c|}
\hline Approach & Interface Component & Modulus (GPa) & Poisson's ratio & Thickness $(\mu \mathrm{m})$ \\
\hline Total etch 3 Steps & Adhesive & 3.6 & 0.28 & 0.5 \\
& Hybrid layer & 2.7 & 0.28 & 4.0 \\
& Tags length Tags & 3.5 & & 30.8 \\
& Base & & & 3.5 \\
& Tags End & & 0.28 & 1.7 \\
\hline Total etch & Adhesive & 1.9 & 0.28 & 0.5 \\
2 Steps & Hybrid layer & 2.1 & & 0.8 \\
& Tags length Tags & 2.5 & & 17.5 \\
& Base & & & 2.5 \\
& Tags End & & 0.28 & 1.3 \\
\hline Self-etch & Adhesive & 3.6 & 0.28 & 0.5 \\
2 Steps & Hybrid layer & 3.7 & & 1.3 \\
& Tags length Tags & 3.4 & & 23.2 \\
& Base & & 0.28 & 2.3 \\
& Tags End & & 0.28 & 1.5 \\
\hline Self-etch & Adhesive & 1.9 & & 0.5 \\
1Step & Hybrid layer & 2.1 & & 14.3 \\
& Tags length Tags & 2.2 & & 2.4 \\
& Base & & & 1.9 \\
\hline
\end{tabular}

At the bottom of the specimen, all nodes located at $6.5 \mathrm{~mm}$ from the adhesive-dentin interface were assumed to be fully constrained to mimic the support span of $13 \mathrm{~mm}$. loading were applied to each model. The load used in each test was the fracture load amount obtained from the experimental work in Newton. The points of application were the two fixtures on the upper surfaces of the specimen at $3.25 \mathrm{~mm}$ from the adhesive-dentin interface to form the loading span of $6.5 \mathrm{~mm}$.

In the post-processing phase, the output of the processing phase was displayed as graphical and numeric outputs in the form of von Mises Stress.

The von Mises and maximum principal stresses of the adhesive layer at the adhesive-dentin interface were analyzed. Von Mises criterion has been chosen as being representative of a multiaxial stress state. The resulting numeric data were evaluated and used to determine the highest stress distribution and where the most probable point of fracture could occur at the corresponding experimental load. The numeric data were transformed into color graphics.

\section{Statistical analysis}

Statistical analysis was performed with IBMSPSS (IBM Company, NY,USA) Statistics Version 25 for Windows. Data were tested for normality and presented as mean and standard deviation (SD) values. One-way analysis of variance ANOVA test followed by post hoc Tukey test was used to compare between more than two groups in non-related samples. The significance level was set at $\mathrm{P} \leq 0.05$.

\section{RESULTS}

\section{Assessment of the fracture toughness of different adhesives-dentin interfaces experimentally:}

The means for fracture toughness value in $\mathrm{MPa}$ $\mathrm{m}^{1 / 2}$ for different groups are shown in (Table 5) and (Figure 5). 
Group I (total etch adhesive 3 step) $1.78 \mathrm{MPam}^{1 / 2}$ and group III (self etch adhesive 2 step) 1.72 MPam $^{1 / 2}$ showed the highest mean values. They were followed by group II (total etch adhesive 2 step) $1.59 \mathrm{MPam}^{1 / 2}$. Whereas the least mean value was recorded in group IV (self etch adhesive 1 step) $1.13 \mathrm{MPam}^{1 / 2}$.

One-way ANOVA test revealed significant difference between groups at $\mathrm{p}<0.001$ level $(\mathrm{p}=$ $0.0001)$. Tukey's post hoc test revealed that there was no significant difference between group I and group III where ( $\mathrm{p}=0.776)$, no significant difference between group II and group III where $(\mathrm{p}=0.189)$.

TABLE (5): Mean and standard deviation (SD) values of fracture toughness in $\left(\mathrm{MPa} \mathrm{m}^{1 / 2}\right)$ for different adhesive systems.

\begin{tabular}{|c|c|c|c|c|}
\hline Variables & Mean & SD & St error & p-value \\
\hline Group I & $1.78^{\mathrm{A}}$ & 0.15 & 0.05 & $\mathbf{0 . 0 0 5}$ \\
\hline Group II & $1.59^{\mathrm{B}}$ & 0.09 & 0.03 & $\mathbf{0 . 1 4 4 N S}$ \\
\hline Group III & $1.72^{\mathrm{AB}}$ & 0.14 & 0.05 & $\mathbf{0 . 0 5 8 N S}$ \\
\hline Group IV & $1.13^{\mathrm{C}}$ & 0.11 & 0.04 & $<\mathbf{0 . 0 0 1 *}$ \\
\hline p-value & \multicolumn{5}{|c|}{$<\mathbf{0 . 0 0 1 *}$} \\
\hline
\end{tabular}

Means with different capital letters in the same row indicate significant difference *; significant $(p<0.05) \mathrm{NS}$; non-significant $(p>0.05)$
Group IV showed a significantly lower value compared to other groups.

\section{Finite element analysis of the dentine side notch} on testing of the different groups:

Dentin and composite were removed from the image to better visualize stress analysis at the adhesive-dentin boundary. The von Mises stress distribution showed the highest stress level at the notch tip, while the lowest stress was found in the middle of the specimen (Figure 6).

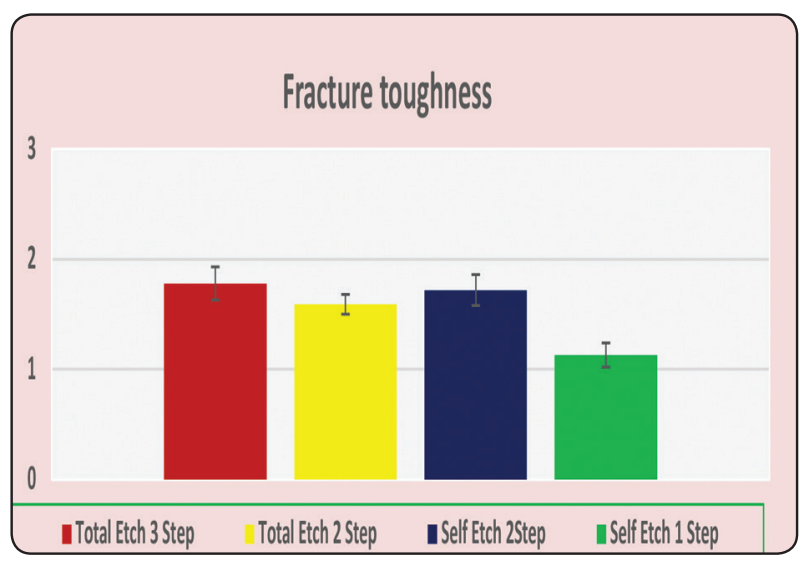

Fig. (5): Column chart showing mean fracture toughness in $\mathrm{MPa}$ m1/2 for different groups

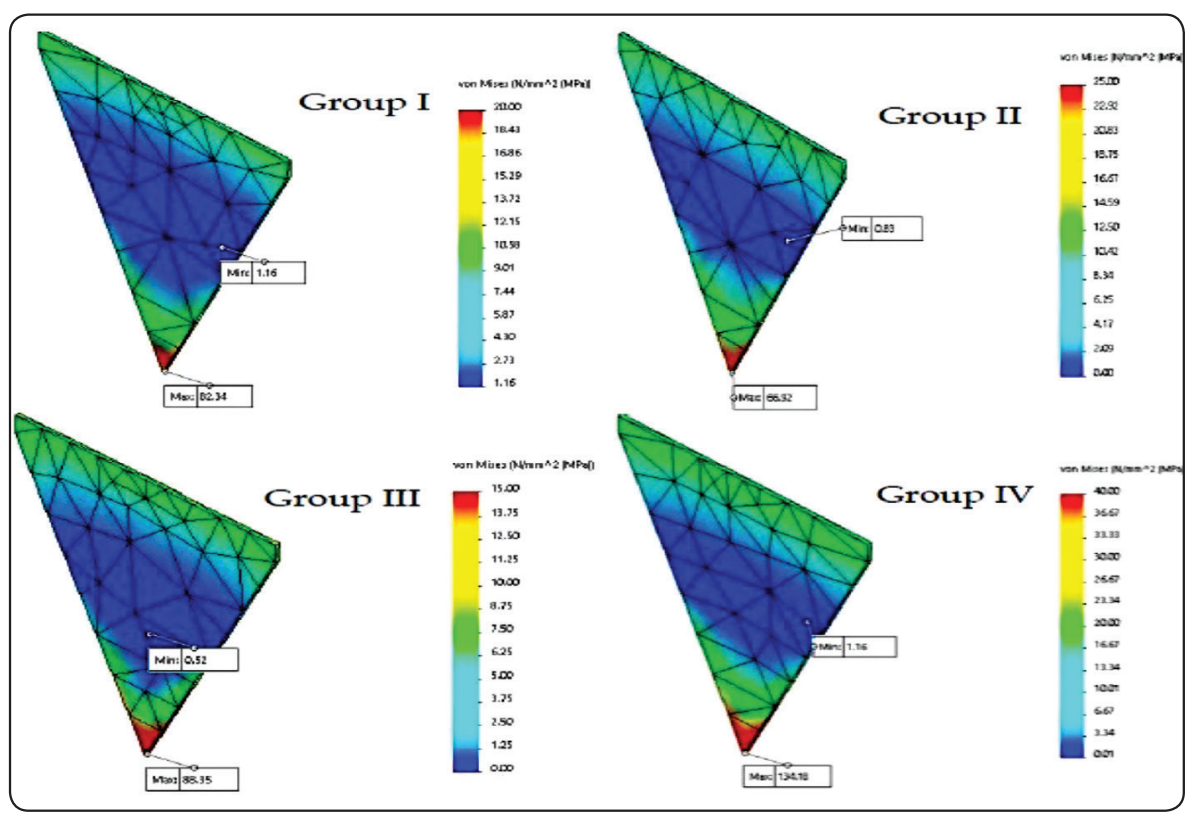

Fig. (6): von Mises stress pattern of the tested groups at the notch tip. 
The Maximum von-Mises stress values for different models induced by the fracture loads obtained from the experimental work shown in the following (Table 6).

TABLE (6): Maximum von Mises stress value in (MPa).

\begin{tabular}{|c|c|}
\hline Models & Maximum Von Mises stress (MPa) \\
\hline Group I & 82.34 \\
\hline Group II & 96.2 \\
\hline Group III & 88.35 \\
\hline Group IV & 134.18 \\
\hline
\end{tabular}

The finite element analysis indicated that the 1-step self etch adhesive model showed the highest maximum Von Mises stress (134.18 MPa) followed by 2 step total etch (96.2 MPa) then 2-step self etch (88.35 MPa) and 3-step total etch (82.34MPa) ,and it was concentrated at the crack of the beams in the hybrid and adhesive layers.

\section{DISCUSSION}

The formation of a dentin-composite resin interface that is resistant to fracture and microleakage is fundamental to almost all restorative adhesive dentistry procedures. Despite recent advances in dentin bonding, this junction is still a critically weak link in a resin composite restoration. ${ }^{(15)}$

Bond strength tests can predict the longevity of a restoration. Dental adhesives are usually tested in shear or tension even though neither of these approaches measures the local stress triggering failure. Because the stress level varies extensively over the bonded surface, it seems as a fracture mechanics approach would be more appropriate way to study dental adhesion. ${ }^{(1)}$

Ideally, bonding effectiveness should be measured by determining the strength of the adhesive interface solely, which can be achieved best using a fracture toughness approach. ${ }^{(16)}$
In this study fracture toughness test was used as its values are not test-dependent and also less affected by random flaws in the test specimens so, better comparable, and less variable and more universal values.

It has some limitations as it is a more laborintensive technique in terms of time, materials and equipment, specimen preparation is difficult, the measurements of interfacial failures are more complicated than similar measurements performed on single component materials, and no standard procedure for dental adhesives is available. However, despite these limitations the fracture mechanics is the proper way to study the dental adhesion. ${ }^{(4)}$

In this study the chevron notch approach has been used as it the most widely used approach when it comes to determining the fracture toughness of dental adhesives. The first fracture toughness study of dentin/composite resin interfaces was done by Tam et al. ${ }^{(17)}$

The ISO 24370 standards to measure fracture toughness of fine ceramics by a chevron-notched beam (CNB) method was applied in the current study. However, some modifications were made during specimen preparation; as the chevron notch produced by placing a spacer on the dentin surface before applying adhesives to make a standardized chevron shaped notch, as cutting the notch induces high residual compressive stress and more prone to microcracks which may act as crack initiators and so lower the fracture toughness values. ${ }^{(18)}$

Other advantage of the present chevron notch beam (CNB) fracture toughness set-up is that it permits the use of common 4-point bend loading to ensures a more uniform and controllable loading. ${ }^{(19)}$

Teeth used were 3 rd molars in the age range of 30 40 years. This is important since dentin decreases in permeability as the age increases and this affects the bond strength, all the dentin specimens were taken 
from the same area of the superficial coronal dentin to allow some standardization. Also the dentin with an intact root is less prone to severe dehydration as thin dentin discs. ${ }^{(20)}$

Very little numerical data are available in literature regarding fracture toughness of adhesives bonded to dentin. On top of that, most data are from older types of adhesives with large variations in bond strength values, mainly due to a lack of standardization of test procedures used in the determination of bond strengths and due to the existence of numerous variables that can influence bond testing. ${ }^{(21)}$

Some difficulties associated with laboratory test methods (fabrication of small specimens, the introduction of sharp crack etc.) led the researchers to a numerical solution. Therefore, the finite element method was applied to fracture mechanics and it is found that it gives satisfactory results when correlated with those obtained experimentally. ${ }^{(22)}$

In this study finite element analysis was used for assessing the stress distribution behavior and measuring the maximum induced stresses by the fracture loads obtained in the experimental work to analyze the von Mises and maximum principal stresses of the adhesive layer at the adhesive-dentin.

Results of the fracture toughness values showed significantly higher mean values for group1 (3 step total etch adhesive). This may be due to the ability of acid etching step to improve the bond strength to dentin. As it solubilize the mineral content of dentin (including the smear layer) to allow the adhesive monomers to infiltrate into the collagen network and replace the water between the collagen fibrils forming a hybrid layer, and in combination with the resin tags inside dentine tubules provides a wide surface for adhesion and allow for micromechanical interlocking. ${ }^{(23,24)}$

Our results are in agreement with De Munck et al (2) results who stated that for both the interfacial fracture toughness and microtensile bond strength test, a general trend could be observed that multistep adhesives bonded more effectively than simplified one-step adhesives. This overall trend was also following the same trend as found in a recent meta-analysis on parameters involved in dentin bonding. ${ }^{(25)}$

Our results are also supported by Alexandra et al (23) who stated that 3step total etch adhesive systems have been shown to perform well in both laboratory and clinical assays and are still seen as the gold standard among bonding systems. ${ }^{(26,27)}$

Our results are also supported also by Ayres et al ${ }^{(28)}$ results they declared that the fracture toughness values appeared to depend on both the adhesive and application mode as multi-mode adhesives performed more effectively in total etch mode than in self-etch mode. ${ }^{(29)}$

The finite element analysis of the Von Mises stress values models showed that the stresses induced by the fracture loads obtained in the experimental work were correlated to the experimental results as 3-step total etch showed the lowest von mises stresses follwed by 2-step self etch and 2-step totel-etch then 1 -step self etch.

All models exhibited similar stress distribution pattern. As the origin of failure which is the chevron notch is placed exactly at the adhesive-dentin interface so, these materials behave in a brittle manner causing the Von Mises stress primarily induced and initiated at the resin-dentin interface.

Crack propagation preferentially occurred along the adhesive-dentin interface, which should be considered a prerequisite to determine the strength of the actual adhesive interface.

The highest maximum von Mises stress which represent the most probable point of fracture could occur at the corresponding experimental load in the adhesive and the hybrid layer. 


\section{CONCLUSIONS}

Within the limitations of this study it could be concluded that:

- Multi-step adhesives showed the highest fracture toughness values than simplified onestep adhesives approach.

- Finite element analysis is an effective tool for fracture toughness evaluation.

\section{Funding sources}

This research did not receive any specific grant from funding agencies in the public, commercial, or not-for-profit sectors

\section{REFERENCE}

1. El Mourad AM. Assessment of Bonding Effectiveness of Adhesive Materials to Tooth Structure using Bond Strength Test Methods: A Review of Literature. The open dentistry journal. 2018;12:664-78.

2. De Munck J, Poitevin A, Luhrs AK, Pongprueksa P, Van Ende A, Van Landuyt KL, et al. Interfacial fracture toughness of aged adhesive-dentin interfaces. Dental materials : official publication of the Academy of Dental Materials. 2015;31(4):462-72.

3. Armstrong S, Geraldeli S, Maia R, Raposo LH, Soares CJ, Yamagawa J. Adhesion to tooth structure: a critical review of "micro" bond strength test methods. Dental materials : official publication of the Academy of Dental Materials. 2010;26(2):e50-62.

4. Soderholm K-J. Review of the fracture toughness approach. Dental Materials. 2010;26(2):e63-e77.

5. Sirisha K, Tanikonda R, Shankar Y R, Kumar P. Validity of bond strength tests: A critical review-Part II. Journal of conservative dentistry : JCD. 2014;17:305-11.

6. Jancar J. Bond strength of five dental adhesives using a fracture mechanics approach. Journal of the mechanical behavior of biomedical materials. 2011;4(3):245-54.

7. Yahyazadehfar M, Ivancik J, Majd H, An B, Zhang D, Arola D. On the Mechanics of Fatigue and Fracture in Teeth. Appl Mech Rev. 2014;66(3):0308031-3080319.

8. Sundar S, Nandlal B, Degala S, Goud M. Finite Element Analysis: A Maxillofacial Surgeon's Perspective. Journal of maxillofacial and oral surgery. 2012;11:206-11.
9. ISO 24370:2005. Fine ceramics (advanced ceramics, advanced technical ceramics) - Test method for fracture toughness of monolithic ceramics at room temperature by chevron-notched beam (CNB) method to measure fracture toughness of ceramics. International Organization for Standardization2005.

10. Anchieta R, Machado L, Martini AP, dos Santos P, Giannini M, Janal M, et al. Effect of long-term storage on nanomechanical and morphological properties of dentinadhesive interfaces. Dental Materials. 2014;31.

11. Anchieta RB, Machado LS, Sundfeld RH, Reis AF, Giannini M, Luersen MA, et al. Effect of partially demineralized dentin beneath the hybrid layer on dentin-adhesive interface micromechanics. Journal of biomechanics. 2015;48(4):701-7.

12. Soares PV, Santos-Filho PCF, Queiroz EC, Araujo TC, Campos RE, Araujo CA, et al. Fracture resistance and stress distribution in endodontically treated maxillary premolars restored with composite resin. Journal of prosthodontics : official journal of the American College of Prosthodontists. 2008;17(2):114-9.

13. Asmussen E, Peutzfeldt A, Sahafi A. Finite element analysis of stresses in endodontically treated, dowel-restored teeth. The Journal of prosthetic dentistry. 2005;94(4):321-9.

14. Anchieta RB, Machado LS, Martini AP, Santos PH, Giannini M, Janal M, et al. Effect of long-term storage on nanomechanical and morphological properties of dentinadhesive interfaces. Dental materials : official publication of the Academy of Dental Materials. 2015;31(2):141-53.

15. Betancourt DE, Baldion PA, Castellanos JE. ResinDentin Bonding Interface: Mechanisms of Degradation and Strategies for Stabilization of the Hybrid Layer. International Journal of Biomaterials. 2019;2019:5268342.

16. Pongprueksa P, Munck J, de Castro Ferreira Barreto B, Karunratanakul K, Van Meerbeek B. Mini-interfacial fracture toughness as a new validated enamel-bonding effectiveness test. Journal of the mechanical behavior of biomedical materials. 2016;62.

17. Far C, Ruse ND. Effect of bleaching on fracture toughness of composite-dentin bonds. The journal of adhesive dentistry. 2003;5(3):175-82.

18. Noort R, Cardew GE, Howard I, Noroozi S. The Effect of Local Interfacial Geometry on the Measurement of the Tensile Bond Strength to Dentin. Journal of dental research. 1991;70:889-93. 
19. Ruse ND, Troczynski T, MacEntee MI, Feduik D. Novel fracture toughness test using a notchless triangular prism (NTP) specimen. Journal of biomedical materials research. 1996;31(4):457-63.

20. Afshar H, Baradaran Nakhjavani Y, Rahro Taban S, Baniameri Z, Nahvi A. Bond Strength of 5(th), 6(th) and 7(th) Generation Bonding Agents to Intracanal Dentin of Primary Teeth. J Dent (Tehran). 2015;12(2):90-8.

21. De Munck J, Mine A, Vivan Cardoso M, De Almeida Neves A, Van Landuyt KL, Poitevin A, et al. Effect of dentin location and long-term water storage on bonding effectiveness of dentin adhesives. Dental materials journal. 2011;30(1):7-13.

22. Aliha MRM, Bahmani A, Akhondi S. Numerical analysis of a new mixed mode I/III fracture test specimen. Engineering Fracture Mechanics. 2015;134:95-110.

23. Vinagre A, Ramos J. Adhesion in Restorative Dentistry. 2016.

24. Cuevas-Suarez CE, da Rosa WLO, Lund RG, da Silva AF, Piva E. Bonding Performance of Universal Adhesives: An Updated Systematic Review and Meta-Analysis. The journal of adhesive dentistry. 2019;21(1):7-26.
25. De Munck J, Mine A, Poitevin A, Van Ende A, Cardoso MV, Van Landuyt KL, et al. Meta-analytical review of parameters involved in dentin bonding. Journal of dental research. 2012;91(4):351-7.

26. Peumans M, Kanumilli P, De Munck J, Van Landuyt K, Lambrechts P, Van Meerbeek B. Clinical effectiveness of contemporary adhesives: a systematic review of current clinical trials. Dental materials : official publication of the Academy of Dental Materials. 2005;21(9):864-81.

27. Heintze SD, Rousson V. Clinical effectiveness of direct class II restorations - a meta-analysis. The journal of adhesive dentistry. 2012;14(5):407-31.

28. Ayres APA, Pongprueksa P, Munck J, Vananroye A, Clasen C, Nascimento F, et al. Mini-interfacial fracture toughness of adhesive-dentin interfaces after plasma pretreatment2016. e73-e4 p.

29. Hanabusa M, Mine A, Kuboki T, Momoi Y, Van Ende A, Van Meerbeek B, et al. Bonding effectiveness of a new 'multi-mode' adhesive to enamel and dentine. Journal of dentistry. 2012;40(6):475-84. 